\title{
Utilisation des lasers excimères dans le domaine du collage des matériaux
}

\author{
P. Laurens, B. Sadras, F. Decobert*, F. Aréfi** et J. Amouroux**
}

Laboratoire d'Application des Lasers de Puissance (LALP), Unité Mixte ETCA-CNRS, 16 bis avenue Prieur de la Côte d'Or, 94114 Arcueil cedex, France

* ETCA/CREA/Polymère et Composite, 16 bis avenue Prieur de la Côte d'Or, 94114 Arcueil cedex, France

** Laboratoire des Réacteurs en Phase Plasma, ENSCP, 11 rue Pierre et Marie Curie, 75231 Paris cedex, France

\begin{abstract}
Résumé : Les modifications de surface induites par un traitement par laser excimère sont susceptibles d'induire une amélioration considérable des propriétés d'adhésion de certains matériaux comme par exemple le polyetherethercétone (PEEK).
\end{abstract}

\section{GENERALITES}

L'assemblage de matériau par collage présente par rapport aux techniques d'assemblage mécanique de nombreux avantages, liés principalement aux réductions de coût de fabrication et aux gains de poids. Néanmoins, la réalisation d'assemblage par collage nécessite généralement une étape préalable de préparation des surfaces à coller afin d'améliorer la tenue mécanique du joint collé et d'en assurer la fiabilité en service. La maîtrise des prétraitements de surface avant collage, qui ont pour objectif de modifier la surface initiale de l'adhérent pour favoriser les interactions interfaciales entre l'adhérent et l'adhésif, constitue donc une étape clé du développement des techniques de collage. Différents types de modifications de surface sont susceptibles d'augmenter "l'affinité " entre l'adhérent et l'adhésif : l'augmentation de la rugosité (ancrage mécanique), l'élimination d'une couche superficielle de faible cohésion (couche de contamination, couche de moindre résistance mécanique, ...), l'augmentation de l'énergie de surface (amélioration du mouillage de la surface par l'adhésif), la modification de la nature chimique de la surface (introduction d'espèces réactives vis à vis de l'adhésif, agent de couplage). La variété des couples "adhérent - adhésif" et des types de modifications favorisant leur assemblage a conduit au développement de multiples techniques de prétraitement de surface avant collage. Celles-çi peuvent être classées en trois catégories principales : techniques mécaniques (nettoyage, abrasion, décapage), techniques par voie chimique (anodisation, décapage chimique, chloruration, organosilane, ajout de primaires, ...), techniques énergétiques (flammage, techniques plasma ou laser). Outre leur efficacité et leur fiabilité vis à vis du collage, ces techniques doivent également répondre à d'autres critères : aspect économique, possibilité d'automatisation et de contrôle du procédé, respect de l'environnement (réduction des effluents et limitation des produits toxiques). La prise en compte de ces critères conduit actuellement à favoriser les efforts dans le domaine des techniques par voie énergétique. C'est ainsi que la recherche et le développement des techniques plasma dans le domaine du collage est un secteur d'activités croissantes depuis plusieurs années. En ce qui concerne les procédés laser, l'intérêt pour cet outil est encore récent et les travaux réalisés peu nombreux. L'essentiel des travaux rapportés dans la littérature utilise des lasers excimère et s'applique principalement aux matériaux organiques (polycarbonate, polyetherimide, polyetherethercétone, polypropylène, téflon) $[1,2,3]$ ou métalliques $[1,4,5]$ (principalement : alliages d'aluminium) mais également à des matériaux céramiques [6,7]. Les atouts d'un procédé de prétraitement avant collage par laser excimère sont multiples : c'est un procédé 
propre, facile à mettre en oeuvre, autorisant un traitement Iocalisé et limité aux zones à assembler ; les profondeurs affectées par le traitement sont faibles $(<1 \mu \mathrm{m})$ et les efrets thermiques très réduits (pas de dégradation du matériau). Par ailleurs, la variété des eftets obtenus sur la surface en fait un procédé pouvant être appliqué à de nombreux matériaux : nettoyage de surface (élimination des contaminants), effets photochimiques ou photothermiques susceptibles d'induire des modifications chimiques de surface favorisant la réactivité de la surface avec l'adhésif, augmentation de la rugosité de surface (régime d'ablation). C'est ainsi que dans le cas de $\mathrm{Si}_{3} \mathrm{~N}_{4}$, l'augmentation importante de la rugosité (formation de cônes) est principalement responsable d'une augmentation de la contrainte à rupture de $45 \mathrm{MPa}$ avec une rupture à l'interface céramique - adhésif (non traité) à $65 \mathrm{MPa}$ (laser $\mathrm{KrF}$ ) avec une rupture cohésive dans l'adhésif [6]. Sur des surfaces métalliques, l'amélioration de la tenue au collage peut résulter d'une augmentation de la rugosité et/ou d'une modification chimique de la surface. Dans le cas d'alliage d'aluminium, l'optimisation des conditions de traitement permet d'augmenter la contrainte à rupture de $2 \mathrm{MPa}$ (non traité) à $14 \mathrm{MPa}[1,8]$; les performances obtenues par laser sont alors supérieures à celle d'un traitement chimique classique d'anodisation ; néanmoins le tenue au vieillissement des joints traités par laser est inférieure à celle obtenue par anodisation. En ce qui concerne les polymères, des augmentations considérables de la tenue au collage peuvent être obtenus aprés traitement par laser excimère; néanmoins la complexité des mécanismes intervenant au cours de l'interaction laser-polymère est telle que la compréhension des mécanismes et des effets obtenus est un sujet d'étude très riche. Nous étudions au laboratoire les potentialités d'un prétraitement avant collage par laser excimère du polyether-ethercétone (PEEK) et du composite PEEK/C (APC2).

\section{DISPOSITIF EXPERIMENTAL ET TECHNIQUES D'ANALYSES}

Le dispositif expérimental est présenté sur la figure 1. Le traitement est réalisé avec un laser excimère Lambda Physics (LPX 200) susceptible de fonctionner à $193 \mathrm{~nm}$ (ArF : $400 \mathrm{~mJ}, 16 \mathrm{~ns}$ ) ou $248 \mathrm{~nm}(\mathrm{KrF}: 600 \mathrm{~mJ}, 26 \mathrm{~ns}$ ). Un dispositif de mise en forme et d'homogenéisation du faisceau permet d'obtenir une zone d'impact carré dont la dimension varie de $5 \times 5 \mathrm{~mm}$ à $15 \times 15 \mathrm{~mm}$ selon la fluence souhaitée sur la cible et dont la répartition en énergie est constante $( \pm 5 \%)$. La fréquence est de $10 \mathrm{~Hz}$. La gamme de fluence utilisée est comprise entre $5 \mathrm{~mJ} / \mathrm{cm}^{2}$ et $250 \mathrm{~mJ} / \mathrm{cm}^{2}$. Le nombre de tirs par unité de surface est compris entre 1 et 2000 . Des tables X-Y assurent le déplacement de l'échantillon sous la cible. Les traitements sont réalisés à pression atmosphérique ; un dispositif de protection gazeuse permet d'effectuer le traitement en atmosphère contrôlée sous argon, azote, air ou oxygène.

Le matériau se présente sous forme de film de PEEK( ${ }^{*}$ ) pur de $250 \mu \mathrm{m}$ dont la cristallinité est de $40 \%$. Des essais ont également été réalisés sur le matériau composite APC2 (résine PEEK/fibre C).

Différentes techniques d'analyse des matériaux ont été utilisées. La profilométrie permet d'obtenir les épaisseurs ablatées en fonction des paramètres de traitement. L'observation des surfaces a été réalisée par microscopie électronique à balayage (MEB : Jeol 6300). La nature chimique des éléments et des liaisons chimiques présentes en surface ont été déterminées par spectroscopie des photoelectrons par rayons X (XPS) (profondeur analysée : $5 \mathrm{~nm}$ ). La spectroscopie par annihilation de positrons (SAP) a permis de déterminer l'épaisseur modifiée par le traitement. L'évolution de la mouillabilité de la surface est suivie par mesure d'angle de contact. Enfin, les propriétés d'adhésion

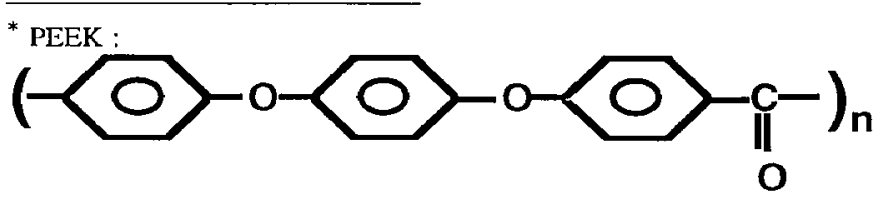


sont obtenues par des tests mécaniques en cisaillement ou en clivage (test DCB) en utilisant deux adhésifs époxy : soit un adhésif époxy supporté (AFM 163) de mise en oeuvre à chaud $\left(120^{\circ} \mathrm{C}, 1 \mathrm{~h}\right)$, soit un adhésif bicomposant (Ciba 2016) de mise en oeuvre à froid. Les surfaces traitées sont maintenues après le traitement 48 h à l'abri de la poussière avant collage.

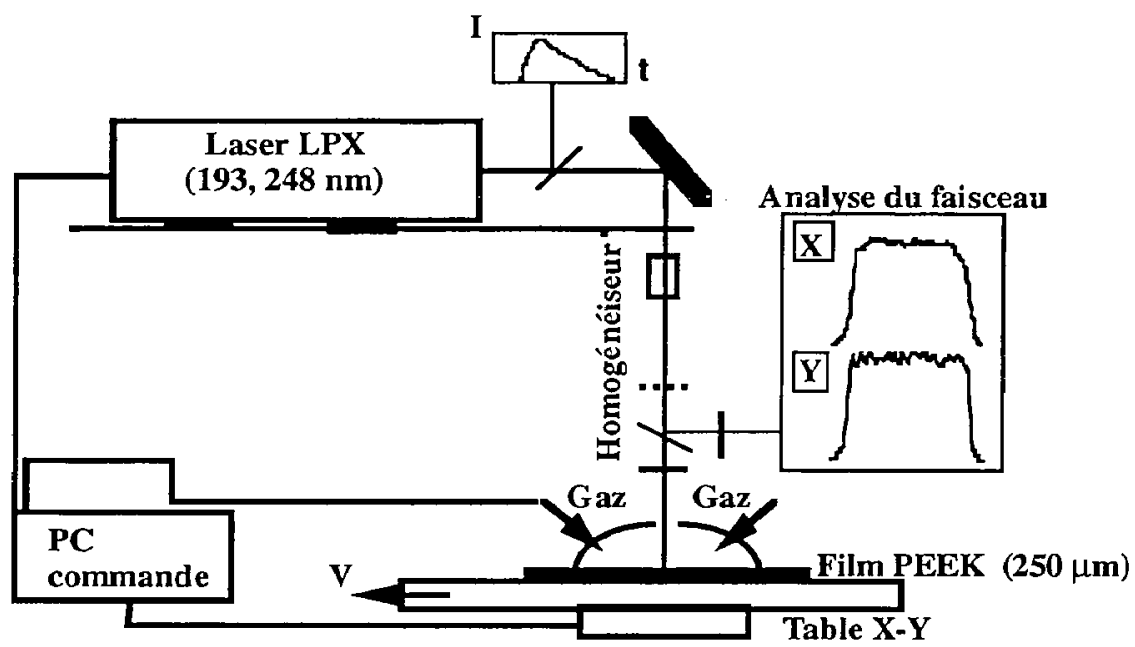

Figure 1 : Dispositif expérimental.

\section{DETERMINATION DES SEUILS D'ABLATION}

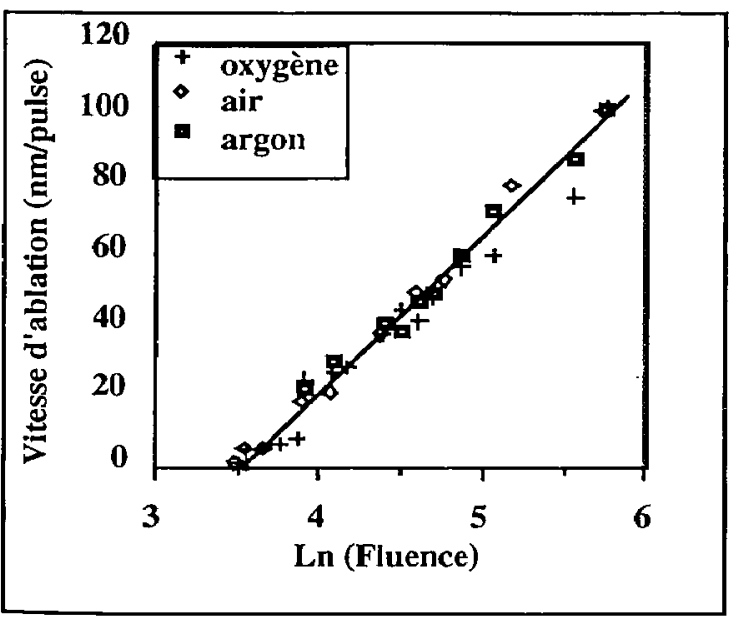

La profilométrie a été utilisée pour déterminer les seuils et vitesses d'ablation à $193 \mathrm{~nm}$ et $248 \mathrm{~nm}$ en fonction de la nature du gaz de traitement. Les seuils d'ablation, $F_{\text {seuil, sont de } 35 \pm 3 \mathrm{~mJ} / \mathrm{cm}^{2} \text { à }}$ $193 \mathrm{~nm}$ et $50 \pm 3 \mathrm{~mJ} / \mathrm{cm}^{2}$ à 248 nm. A $193 \mathrm{~nm}$, l'Evolution des vitesses d'ablation en fonction de la fluence suit une loi de Beer Lambert $\left(\mathrm{d}=1 / \alpha \operatorname{Ln}\left(\mathrm{F} / \mathrm{F}_{\text {seuil }}\right)\right.$ dans la gamme de fluence étudiée et est indépendante de la nature du gaz de protection. (Fig.2).Les coefficients d'absorption valent $2.1510^{5} \mathrm{~cm}^{-1}$ à $193 \mathrm{~nm}$ et 1.5 $10^{5} \mathrm{~cm}^{-1}$ à $248 \mathrm{~nm}$.

Figure 2 : Vitesse d'ablation en fonction de la fluence à $193 \mathrm{~nm}$ sous différentes atmosphères gazeuses.

\section{CARACTERISATION DES SURFACES TRAITEES}

Les traitements ont été réalisés d'une part en régime de traitement de surface $\left(F<F_{\text {seuil }}\right)$ et d'autre part en régime d'ablation $\left(\left(\mathrm{F}>\mathrm{F}_{\text {seuil }}\right)\right.$. Dans le régime de traitement de surface, 
les modifications de surface du PEEK traité ont été étudiées en fonction du nombre de tirs par unité de surface, de la fluence et de la nature de l'atmosphère đe traitement. En régime d'ablation, nous avons suivi l'évolution des surfaces traitées en fonction de la fluence.

\subsection{Régime d'ablation}

Le traitement en régime d'ablation conduit à la formation sur la surface traitée d'un dêpôt noir dont une partie peut être éliminée par nettoyage ultérieur aux ultra sons de la surface. L'analyse de ce dépôt par spectroscopie infra rouge montre qu'il est principalement constitué de carbone élémentaire. L'analyse XPS des surfaces traitées à $193 \mathrm{~nm}$ ou 248 nm met en évidence que la surface présente un fort enrichissement en carbone par rapport au matériau non traité. L'augmentation de la fluence tend à réduire l'importance de ce đépôt en surface et à diminuer l'enrichissement en carbone de de la surface (traitement à $248 \mathrm{~nm}, 80 \mathrm{~mJ} / \mathrm{cm} 2, \mathrm{O} / \mathrm{C}=0.03 ; 250 \mathrm{~mJ} / \mathrm{cm} 2, \mathrm{O} / \mathrm{C}=0.09 ;$ matériau non traité : $\mathrm{O} / \mathrm{C}=$ 0.20 ). La présence d'un redépôt riche en carbone a été observée sur de nombreux polymères (PI, PET) $[[9,10]$. Ce dépôt est responsable de l'apparition d'une forte rugosité (cônes) des surfaces de PEEK traitées en régime d'ablation pour des fluences

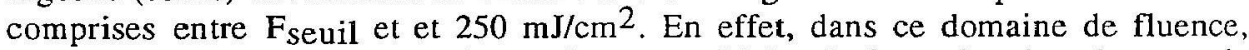
l'élimination du redépôt entre chaque tir permet d'éviter la formation des cônes sur la surface (Fig.3). La formation de ces cônes est attribuée à un effet d'écran des redépôts dont la forte teneur en carbone tend à augmenter le seuil d'ablation. A forte fluence, aucun redépôt, ni cône ne sont plus observés au MEB sur la surface. Ceci résulte probablement d'une dégradation plus complète des fragments ablatés dans le plasma d'ablation.

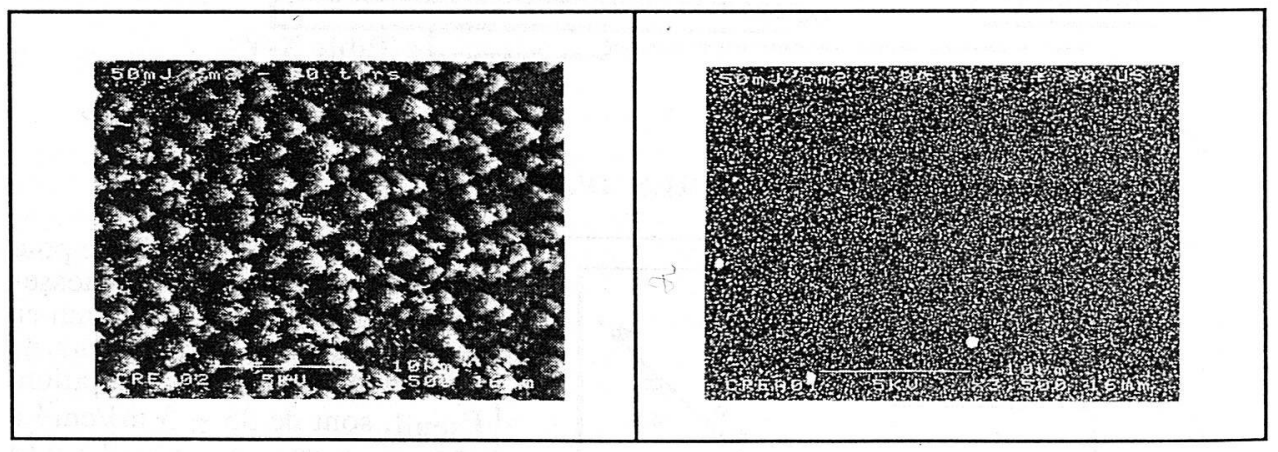

Figure 3 : Morphologie de surface (MEB) aprés traitement par laser excimère en régime d'ablation (50 $\mathrm{mJ} / \mathrm{cm}^{2}, 80$ tirs, air). A gauche : sans nettoyage de la surface entre chaque tir, à droite nettoyage de surface aux ultra sons entre chaque tir.

\subsection{Régime de traitement de surface}

L'observation au MEB des surfaces traitées fait apparaitre une modification de l'aspect de la surface (Fig.4). En effet, l'observation de la surface non traitée montre une hétérogénéité de la surface liée à la nature semicristalline du matériau initial (présence de sphérulites). Après traitement, l'aspect de la surface est lisse et homogène ; les sphérulites ne sont plus visibles. Il apparaît donc que le traitement induit une élimination des zones cristallines du matériau résultant soit d'une dégradation du matériau, soit d'une amorphisation de celui ci. L'amorphisation d'une surface organique a déjà été mise en évidence dans le cas du traitement du PET par laser excimère [11]. Les épaisseurs des couches modifiées par le traitement laser en régime de traitement de surface ont pu être estimées par spectroscopie d'annihilation des positrons[12]. Pour un traitement réalisé à $20 \mathrm{~mJ} / \mathrm{cm} 2$ en ArF, l'épaisseur modifiée, déterminée par l'expérience, est de l'ordre de $100 \mathrm{~nm}$; cette valeur est trés proche de l'estimation de la profondeur thermiquement affectee par le traitement, $\mathrm{l}=2(\mathrm{Dt})^{1 / 2}=93 \mathrm{~nm}$ (D : diffusivité thermique). La profondeur 
modifée est indépenclante de la nature du gaz de traitement et du nombre de tirs (entre $2(\kappa)$ et 1000 tirs).

L'analyse par XPS des surfaces traitées a permis de d'étudier l'influence des paramètres du traitement sur la composition chimique de la surface (nombre de tirs, nature de l'atmosphère gazeuse, fluence et fréquence de tirs).

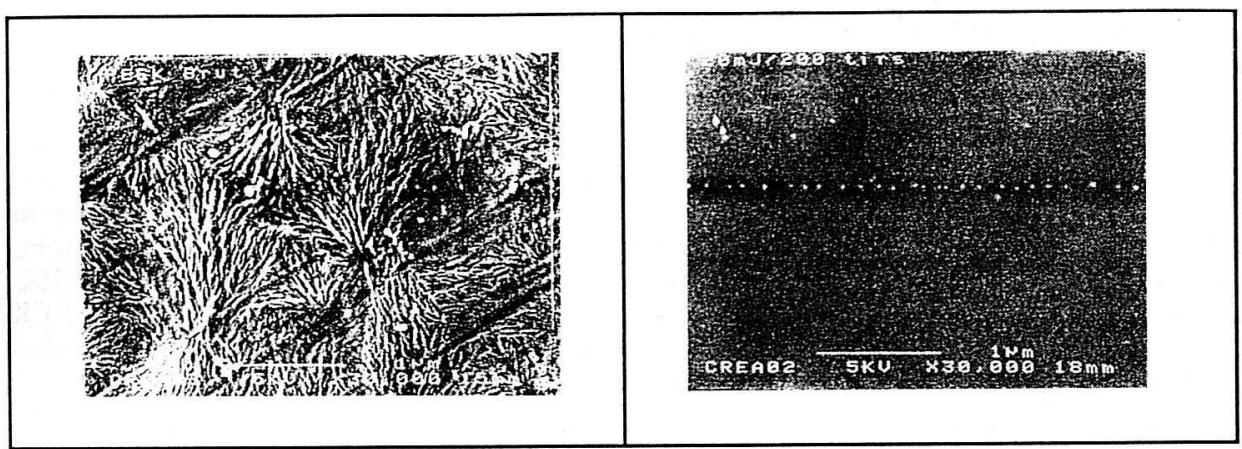

Figure 4 : Morphologie de la surface (MEB) : (a) avant traitement, (b) après traitement en régime de traitement de surface $\left(20 \mathrm{~mJ} / \mathrm{cm}^{2}, 200\right.$ tirs $)$.

L'Évolution de la composition chimique de la surface en fonction du nombre de tirs fait apparaitre deux domaines :

- en début de traitement (nombre de tirs compris entre 1 et 30), le traitement induit principalement un nettoyage de la surface présentant initialement une contamination organique (Tableau 1).

\begin{tabular}{|l|c|c|c|c|c|}
\hline & $\mathbf{O}_{1 \mathbf{s}} / \mathbf{C}_{1 \mathbf{s}}$ & \multicolumn{4}{|c|}{$\mathrm{C}_{1 \mathbf{s}}$} \\
\hline Energie de liaison (eV) & & $\begin{array}{c}\mathbf{2 8 5} \\
(\mathbf{C}-\mathbf{C})\end{array}$ & $\begin{array}{c}\mathbf{2 8 6 . 3} \\
(\mathbf{C}-\mathbf{O})\end{array}$ & $\begin{array}{c}\mathbf{2 8 7 . 3} \\
(\mathbf{C}=\mathbf{O})\end{array}$ & $\begin{array}{c}\mathbf{2 8 8} \\
(\mathbf{O}-\mathbf{C}=\mathbf{O})\end{array}$ \\
\hline Avant traitement & 0.20 & 0.83 & 0.12 & 0 & 0.63 \\
\hline Aprés traitement (a) & 0.16 & 0.73 & 0.25 & 0.04 & 0.01 \\
\hline Référence [13] & 0.158 & 0.74 & 0.21 & 0.05 & 0 \\
\hline
\end{tabular}

(a) : Traitement : $193 \mathrm{~nm}, 20 \mathrm{~mJ} / \mathrm{cm}^{2}, 6$ tirs, argon fréquence : $10 \mathrm{~Hz}$.

Tableau 1 : Analyse XPS - Nature et teneur relative des différentes liaisons chimiques présentes en surface

- pour un nombre de tirs supérieur, le traitement induit des modifications chimiques de la surface de PEEK. La nature et l'importance de ces modifications dépendent essentiellement du nombre de tirs et de l'atmosphère de traitement mais sont peu sensibles aux variations de la fluence $\left(F: 5 \mathrm{~mJ} / \mathrm{cm}^{2}-30 \mathrm{~mJ} / \mathrm{cm}^{2}\right)$ ou de la frequence de tirs (f : $1-20 \mathrm{~Hz}$ ).

Les spectres XPS du carbone $\left(C_{1 s}\right)$ montrent, quelle que soit la nature du gaz de traitement, une diminution du signal du shake up (292 eV) avec l'augmentation du nombre de tirs. Ceci met en évidence une rupture des cycles aromatiques au cours de traitement (PEEK pur : shake up $/ \mathrm{C}=0.045$; traitement sous air, $20 \mathrm{~mJ} / \mathrm{cm}^{2}: 200$ tirs : shake up $/ \mathrm{C}=0.023 ; 2000$ tirs : shake up/C $=0.015$ ). Par ailleurs, on observe, la formation d'une nouvelle liaison , $\mathrm{O}-\mathrm{C}=\mathrm{O}$, dont la concentration relative sur la surface dépend des conditions de traitement. 


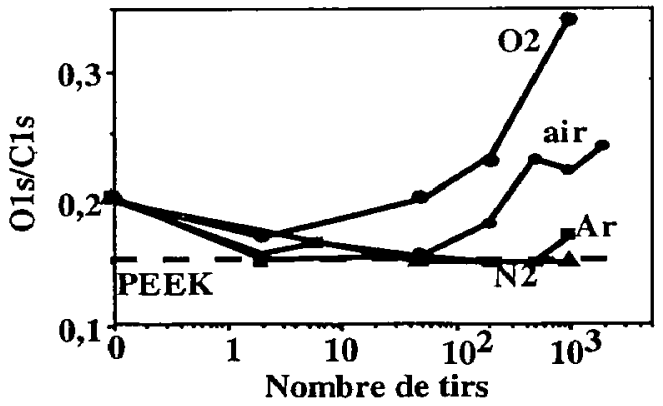

Les évolutions des rapport $\mathrm{O}_{1 \mathrm{~s}} / \mathrm{C}_{1} \mathrm{~s}$ en fonction du nombre de tirs pour les différentes atmosphères de traitement étudiées sont présentées sur la figure 5. En présence d'oxygène dans le milieu gazeux, le traitement induit une oxydation de surface de PEEK; un traitement en atmosphère d'argon ou d'azote ne modifie pas le rapport $\mathrm{O} / \mathrm{C}$ du $\mathrm{PEEK}$. L'évolution des concentrations relatives des différentes liaisons chimiques sur la surface selon l'atmosphère de traitement est présentée dans le tableau 2.

\begin{tabular}{|l|c|c|c|c|}
\hline Traitement laser & $\mathbf{C - C}$ & $\mathbf{C - O}$ & $\mathbf{C = O}$ & $\mathbf{O}-\mathbf{C}=\mathbf{O}$ \\
\hline Argon, 200 tirs & 0.85 & 0.10 & 0.034 & 0.020 \\
\hline Argon,1000 tirs & 0.85 & 0.10 & 0.037 & 0.023 \\
\hline Air, 200 tirs & 0.79 & 0.17 & 0.066 & 0.036 \\
\hline Air, 1000 tirs & 0.78 & 0.15 & 0.066 & 0.063 \\
\hline O2, 200 tirs & 0.76 & 0.18 & 0 & 0.043 \\
\hline O2, 1000 tirs & 0.69 & 0.20 & 0 & 0.110 \\
\hline
\end{tabular}

Tableau 2 : Teneurs relatives des différentes liaisons chimiques présentes en surface (traitement : $193 \mathrm{~nm}, 20 \mathrm{~mJ} / \mathrm{cm}^{2}$ )

On constate que la nature de l'atmophère de traitement a une influence significative sur la teneur relatuve des différentes liaisons chimiques présentes en surface après traitement. En effet, lorsque la teneur en oxygène du milieu gazeux décroit, les rapports de liaisons carbone-carbone augmente et les teneurs en liaisons $\mathrm{C}-\mathrm{O}$ et $\mathrm{O}-\mathrm{C}=\mathrm{O}$ diminuent.

L'ensemble de ces résultats suggèrent que le traitement laser induit des ruptures de liaisons au niveau des cycles aromatiques, des groupements $\mathrm{C}-\mathrm{O}-\mathrm{C}$ et $\mathrm{C}-\mathrm{C}=\mathrm{O}$. Les radicaux formes se recombinent principalement sous forme de liaisons carbone-carbone en atmosphère neutre ou réagissent avec l'oxygène gazeux pour former des groupements $\mathrm{C}-\mathrm{OH}$ et $\mathrm{COOH}$.

L'ensemble des modifications observées ont une influence favorable sur les propriétés de mouillabilité de la surface. L'angle de contact de la surface initiale, $\theta$, est de $80^{\circ}$; le traitement induit une diminution des angles de contact (augmentation de la mouillabilité de surface) plus marquée lorsque le traitement est réalisé sous oxygène (traitement ArF, $20 \mathrm{~mJ} / \mathrm{cm}^{2}, 200$ tirs : argon : $\theta=75^{\circ}$, air : $\theta=68^{\circ}$, oxygène : $\theta=63^{\circ}$ ).

\subsection{Propriétés d'adhésion}

Les propriétés d'adhésion des substrats avant et après traitement ont été caractérisées en utilisant des tests de sollicitations en cisaillement et en clivage (test DCB). La comparaison des résultats obtenus avec ces deux tests a tout d'abord clairement mis en évidence une meilleure sélectivité du test en clivage par rapport au test de cisaillement. En effet, si le test en cisaillement montre une augmentation importante de la contrainte à rupture après traitement laser ( $45 \pm 5 \mathrm{MPa}$, rupture cohésive dans l'adhésif (AFM 163)) par rapport au substrat non traité (18 $\mathrm{MPa}$, rupture adhésive à l'interface PEEK-adhésif), il ne permet pas d'observer de variation des contraintes à rupture (ou des faciès de 
rupture) Iorsque les conditions de traitement laser varient. Ce test ne permet pas non plus de différencier l'efficacité d'un traitement laser de celle obtenue par un traitement en décharge couronne, alors que les résultats obtenus par le test en clivage montrent que les énergies et les faciès de rupture obtenus après traitement laser ou traitement en décharge couronnesont très différents : après traitement laser, on observe une rupture cohésive dans l'adhésif avec une contrainte à rupture de $750 \mathrm{~J} / \mathrm{m}^{2}$; le traitement par décharge couronne conduit à une rupture localisée à l'interface PEEK/adhésif, l'énergie de rupture est de $20 \mathrm{~J} / \mathrm{m}^{2}$. Les résultats obtenus pour différentes conditions de traitement laser à 193 $\mathrm{nm}$ en utilisant le test de clivage sont présentés dans le tableau 3. Les résultats montrent une augmentation très importante des propriétés d'adhésion après traitement par laser excimère. Par ailleurs, on observe pour les traitements réalisés en régime de traitement de surface, l'efficacité supérieure d'un traitement sous argon (ou sous air) par rapport à un traitement sous oxygène. La comparaison des résultats obtenus sous air en régimes de traitement de surface et d'ablation met en évidence une légère supériorité du traitement en régime d'ablation.

\begin{tabular}{|c|c|c|}
\hline \multirow[b]{2}{*}{ Traitement } & \multicolumn{2}{|c|}{ Energie de rupture $\left(\mathrm{J} / \mathrm{m}^{2}\right)$} \\
\hline & Adhésif AFM 163 & Adhésif Ciba 2016 \\
\hline Nettoyage ultra sons & $200 \pm 150(\mathrm{~A})$ & $4 \pm 1(\mathrm{~A})$ \\
\hline Décharge couronne & - & $70 \pm 35(\mathrm{~A})$ \\
\hline $\begin{array}{l}\text { Laser ArF, } 20 \mathrm{~mJ} / \mathrm{cm} 2 \text {, } \\
\text { argon, } 200 \text { tirs }\end{array}$ & $2000 \pm 200(\mathrm{C})$ & $720 \pm 230(\mathrm{C})$ \\
\hline $\begin{array}{l}\text { Laser ArF, } 20 \mathrm{~mJ} / \mathrm{cm} 2 \text {, air, } \\
200 \text { tirs }\end{array}$ & $1500 \pm 250(\mathrm{C})$ & $710 \pm 210(\mathrm{C})$ \\
\hline $\begin{array}{l}\text { Laser ArF, } 20 \mathrm{~mJ} / \mathrm{cm} 2 \text {, } \\
\text { oxygène, } 200 \text { tirs }\end{array}$ & - & $330 \pm 100(\mathrm{C})$ \\
\hline $\begin{array}{l}\text { Laser ArF, } 180 \mathrm{~mJ} / \mathrm{cm} 2 \text {, } \\
\text { air, } 200 \text { tirs }\end{array}$ & $2100 \pm 250(\mathrm{C})$ & $850 \pm 210(C)$ \\
\hline
\end{tabular}

(A) : Rupture adhésive à l'interface PEEK-Adhésif

(C) : Rupture cohésive dans l'adhésif et dans le PEEK

Tableau 3 : Test de clivage : Energie de rupture des joints collés selon la nature du prétraitement.

La corrélation entre les tests mécaniques d'adhésion et l'analyse des modifications observées sur les surfaces traitées ne permet pas actuellement de déterminer clairement la nature des modifications majoritairement responsables de l'amélioration considérable des propriétés d'adhésion du PEEK après traitement par laser excimère à $193 \mathrm{~nm}$. Plusieurs phénomènes ont été mis en évidence : effet de nettoyage de surface, élimination de la cristallinité du matériau initiale, rupture des cycles aromatiques, ruptures de liaisons $\mathrm{C}-\mathrm{O}$, $\mathrm{C}-\mathrm{C}=\mathrm{O}$, formation de groupements $\mathrm{O}-\mathrm{C}=\mathrm{O}$, oxydation du matériau, augmentation de la rugosité (régime d'ablation). L'ensemble de ces modifications sont globalement favorables à l'amélioration de la tenue à l'adhésion, à l'exception d'un effet d'oxydation qui tend à réduire l'efficacité d'un traitement sous oxygène, en entrainant probablement une dégradation importante de surface. On peut supposer que l'efficacité du traitement laser soit liée principalement aux ruptures des liaisons $\mathrm{C}-\mathrm{O}-\mathrm{C}$ et $\mathrm{C}-\mathrm{C}=\mathrm{O}$ trés localisées en surface (épaisseur affectée : $100 \mathrm{~nm}$ ) permettant de libérer les groupements polaires $\mathrm{C}-\mathrm{OH}$ et $\mathrm{COOH}$ susceptibles de réagir avec l'adhésif sans modifier en sous couche les propriétés de cohésion du matériau. Par ailleurs, ces ruptures de chaines en surface sont également susceptibles de favoriser l'interdiffusion à l'interface entre le polymère et l'adhésif.

\section{CONCLUSION}

Les effets induits par un traitement par laser excimère à $193 \mathrm{~nm}$ se sont révélés particulièrement bien adaptés à l'amélioration des propriétés d'adhésion du polyetherethercétone (PEEK). Ce traitement constitue donc pour ce matériau réputé diffícile à assembler une possibilité de traitement "propre", efficace,et relativement facile à mettre 
ocuvre (traitement à pression atmosphérique, sous air). Par ailleurs, l'eflicacité du traitement à fluence inférieure au seuil d'ablation permet de travailler à fluence modérée et donc d'utiliser les sources excimère actuellement disponibles sur le marché. Une étude menée sur le matériau composite PEEK/fibre de carbone, utilisé dans le secteur aéronautique a confirmé l'intérêt et les potentialités du traitement laser puisque la comparaison des performances obtenus par laser sont supérieures à celle obtenus avec les techniques classiques utilisées pour ce matériau (traitement par voie chimique ou traitement par plasma) [14]. En ce qui concerne la compréhension des effets induits sur la surface par le traitement, nous avons mis en $\epsilon$ vidence plusieurs types de modifications toutes susceptibles à priori d'influencer favorablement la tenue au collage du matériau : nettoyage de surface, élimination de la cristallinité du matériau non traité, rupture des cycles aromatiques, des liaisons $\mathrm{C}-\mathrm{O}-\mathrm{C}$ et $\mathrm{C}-\mathrm{C}=\mathrm{O}$ permettant de créer en surface des liaisons polaires susceptibles de favoriser les intéractions entre l'adhésif et le PEEK. La comparaison des effets et résultats obtenus d'une part par laser excimère à d'autre longueurs d'onde $(248 \mathrm{~nm}$ et $308 \mathrm{~nm})$ ainsi qu'en utilisant des traitements par lampe UV devraient permettre d'améliorer la compréhension des effets induits sur la surface.

\section{Remerciements.}

Les auteurs remercient Y. Saliou (ETCA/PC) pour la réalisation des tests d'adhésion.

\section{Références}

[1] A. Buchman, H. Dodiuk, M. Rotel et J. Zahavi, Int. J. Adhesion and Adhesives, 11 (1991) 144-149.

[2] J. Breuer, S. Metev, G. Sepold, G. Kruger, O.D. Hennemann, "Influencing adherence properties of polymers by excimer laser radiation", Excimer Lasers and Applications III, SPIE vol. 1503 (1991) p. 223-230.

[3] M. Murahara, M. Okoshi, K. Toyoda, "Excimer laser induced photochemical defluoruration and substitution of functionnal group onto fluororesin surface for adhesion", Laser Materials Processing : Industrial and Microelectronics Applications" , Vienna (Austria) Avril 1994, SPIE Vol. 2207, p. 577-584.

[4] F. Henari, W. Blau, Applied Optics, 34, (1995) p. 581-584.

[5] Z. Gendler, A. Rosen, M. Bamberger, M. Rotal, J. Zahavi, A. Buchman, H. Dodiuk, J. Mater. Sci., 29 (1994) p. 1521-1525.

[6] T. Ooie, T. Yano, M. Yoneda, M. Katsumura, "New bonding method of metals and ceramics using laser ablation", Laser Materials Processing (ICALEO'95), San Diego (USA), Novembre 1995, published by Laser Institute of America, Vol 80 (1996) p. 441448.

[7] H.C. Man, X.M. Zhang, T.M. Yue, W.S. Lau, "Modification of ceramic surfaces by excimer laser for adhesive bonding", Laser Materials Processing (ICALEO'95), San Diego (USA), Novembre 1995, published by Laser Institute of America, Vol 80 1996) p. 449-458.

[8] H. Dodiuk, A. Buchman, S. Kenig, M. Rotel et J. Zahavi, T. Reinhart, J. Adhesion; 41 (1993) p. 93-112.

[9] R.S. Taylor, K.E. Leopold, D.L. Singleton, G. Paraskevopoulos, R.S. Irwin, J. Appl. Phys., 64 (1988) p. 2815-2818.

[10] S. Kuper, J. Brannon, J. Appl. Phys., 60 (1992) p. 1633-1635.

[11] S. Lazare, P. Benet, J. Applied Physics, 74 (1993) p. 4953-4957.

[12] R.I. Grynszpan, S. Kuna, P.G. Coleman, B. Sadras, P. Laurens, F. Aréfi, J. Amouroux, "Surface analysis by slow positrons of excimer laser treated poly(ether etherketone)", Proceedings of EURHAD'96, Cambridge (GB) Septembre 1996.

[13] C. Jama, O. Dessaux, P. Goudmand, L. Gengembre, J. Grimblot, Surf. and Interface Anal., 18 (1992) p. 751-756.

[14] F. Decobert, B. Saliou, B. Sadras, P. Laurens, Intern. J. of Adhesion and Adhesives (en préparation). 